\title{
Un ciclo de mejora en el aula aplicado en la asignatura de Análisis de Artefactos
}

\section{An improvement cycle in classroom applied in the subject Analysis of Artifacts}

CARLOS P. ODRIOZOLA

ORCID: https://orcid.org/0000-0002-0093-2129

Universidad de Sevilla. Departamento

de Prehistoria y Arqueología

codriozola@us.es

Fecha de recepción: 03-06-2019

Fecha de aceptación: 10-06-2019

DOI: http://dx.doi.org/10.12795/9788447221912.018

Pp.: 424-448 


\section{Resumen}

En la presente publicación se detallan las características del Ciclo de Mejora en el Aula (CIMA) aplicado a la asignatura Análisis de Artefactos, del Grado en Arqueología durante el curso académico 2018-2019. El ciclo de mejora ha consistido en la aplicación de un modelo metodológico centrado en el estudiante que practica una visión constructivista y evolutiva del conocimiento, donde el estudiante se sitúa en el centro del proceso trabajando en la propia construcción del aprendizaje. En las siguientes páginas se expone el modelo ideal y se comentan los problemas, soluciones y mejoras a aplicar en próximos cursos.

Palabras clave: Análisis de artefactos; Grado en Arqueología; Docencia universitaria; Experimentación docente universitaria; Aprendizaje basado en problemas.

\section{Abstract}

The details of the Improvement Cycle in Classroom (ICIC) applied during the academic year 2018-2019 to the subject Analysis of Artifacts, in the Degree in Archeology, are shown in this publication. The Improvement Cycle has been based on the application of a constructivist and evolutionary framework focused on the student as the center of the learning process. The following pages show the ideal model and discuss the problems, solutions and improvements to be applied in future courses.

Keywords: Artifact analysis; Degree in Archaeology; Degree in Criminology; University teaching; University teaching experience; Problem-based learning.

Jornadas de Formación e Innovación Docente del Profesorado | № 2 (2019) Esta obra se distribuye con la licencia Creative Commons Reconocimiento-NoComercial-SinObraDerivada 4.0 Internacional (CC BY-NC-ND 4.0.) 


\section{Contexto}

El Ciclo de Mejora en el Aula (CIMA), realizado como parte del proyecto de innovación docente, se ha aplicado a la asignatura obligatoria de 4 o curso del Grado en Arqueología de la Universidad de Sevilla "Análisis de Artefactos" (código 2420083). La asignatura se imparte entre tres Departamentos a razón de 2 ECTS por departamento: Cristalografía, Mineralogía y Química Agrícola (Facultad de Química), Física aplicada (ETS de Ingeniería) y Prehistoria y Arqueología (Facultad de Geografia e Historia, que coordina la asignatura).

Las clases se han impartido en el Aula XXV de la Facultad de Geografía e Historia durante el segundo cuatrimestre del curso 2018-2019. Un espacio pequeño configurado para 35 alumnos de una manera singular; por un lado, encontramos dispuestas en $\mathrm{U}$ y cubriendo el perimetro del aula una serie de sillas individuales, y por otro un conjunto de pupitres configurados en hileras que miran a la pantalla de proyección. Una configuración pobre y poco dinámica que imposibilita disponer el mobiliario de ninguna otra manera. La mesa del profesor se encuentra a la misma altura que la de los alumnos, de tal forma que si se proyecta algo y el docente se encuentra sentado, éste interrumpe la visión de gran parte del alumnado. Si no se proyecta, el profesor es invisible para la mayoría de los alumnos. La asistencia en general es alta, suelen asistir a clase unos 27-28 alumnos, de 32 que están matriculados.

Llevo impartiendo esta asignatura desde que se implantó en el curso 2016-2017 en el Grado de Arqueología. Los contenidos de esta asignatura fueron desarrollados inicialmente por acuerdo entre los profesores que íbamos a impartirla en base a un consenso amplio. Dividimos los contenidos en bloques que tratan de forma temática diferentes categorías de artefactos y las técnicas instrumentales adecuadas para su análisis. La asignatura 
es interdisciplinar, como no podría ser de otra manera cuando se trata de una materia que trata técnicas instrumentales en un Grado de Humanidades. En ningún curso de los que he impartido docencia en esta asignatura he dejado de tener una sensación de amargura, casi de frustración, ya que los alumnos nunca alcanzaban mis expectativas en cuanto al nivel de aprendizaje. Para los alumnos supone una asignatura diferente a lo que han visto en los tres años anteriores. A pocos meses de licenciarse se encuentran con una asignatura en la que se les exige que aprendan Química y Física, lo que sin duda les provoca una tasa de estrés elevado ante la inseguridad de si serán capaces de superar una asignatura que requiere que aprendan una materia que en muchos casos no han cursado desde primaria y con la que no se sienten cómodos. Este problema no tiene solución ya que es casi imposible que se modifique la estructura del grado o los contenidos que tienen que aprender los alumnos, pero lo que sí me propongo como objetivo es que los alumnos disfruten aprendiendo estos contenidos.

Para poder realizar el CIMA lo primero que me planteo es comprender qué se exige que aprendan los alumnos, para a partir de este punto diseñar unos contenidos adecuados para que logren los resultados del aprendizaje que consigna la Memoria de Verificación del Grado en Arqueología.

\section{¿Qué enseñar?}

Según se describe en el programa de la asignatura del curso académico 2018-2019 la asignatura a nivel conceptual y procedimental se centra en la aplicación de técnicas y métodos procedentes de las Ciencias Naturales, de la Tierra, de Materiales... al análisis de artefactos arqueológicos y cómo la aplicación de estas técnicas se usan en Arqueología para inferir cuestiones acerca de la 
transferencia tecnológica, el comercio / intercambio, la innovación, la identidad ... Es decir, cómo se articula la relación entre la evidencia empírica (cuantificación de determinados aspectos de la cultura material, características fisicoquímicas) y las dimensiones de la Arqueología: espacio, tiempo y forma. Mientras que a nivel actitudinal la asignatura entrena destrezas como el razonamiento crítico acerca de las principales técnicas de laboratorio empleadas en la investigación arqueológica, sus potenciales, limitaciones, protocolos y mejores prácticas. Y a nivel aptitudinal la asignatura pretende cubrir el vacío existente entre Arqueología y Ciencia a través del desarrollo de competencias para el diseño y desarrollo de proyectos arqueológicos basados en técnicas de laboratorio, así como la capacidad de evaluar críticamente el trabajo de otros.

Para ello el profesorado de la asignatura ha diseñado un programa docente para el curso 2018-2019 que persigue alcanzar los siguientes objetivos:

1. Conocer los principios básicos de algunas técnicas de análisis, terminología utilizada y su aplicación al material arqueológico.

2. Familiarizar al alumnado en el proceso completo de análisis de artefactos: preparación de muestras, calibración del instrumental, obtención e interpretación de estos.

3. Primera toma de contacto con algunos de los equipos de análisis más comunes.

4. Proporcionar al alumnado una visión amplia y atractiva del papel que juega el análisis de artefactos en la Arqueología moderna.

5. Acercar al alumnado a los debates actuales sobre la adquisición de datos, análisis e interpretación.

Estos objetivos docentes han sido diseñados para dar cumplimiento a su vez a los resultados del aprendizaje que se consignan en la Memoria de Verificación del Grado: 
1. Conocimiento de los métodos y técnicas de obtención y elaboración de datos.

2. Desarrollar una buena percepción del concepto de analítica y su alcance.

3. Conocimiento de los fundamentos de las técnicas fisicoquímicas utilizadas en Arqueología.

4. Aprender la correcta lectura e interpretación de los resultados de estas analíticas.

\section{Diseño del Ciclo de Mejora en el Aula}

Una vez hemos analizado lo que debemos enseñar vamos a diseñar un CIMA para la asignatura. En este caso el ciclo se realizará -como se ha dicho- sobre 20 horas, es decir, el total de los ECTS que imparto en la asignatura. Estos 2 ECTS se estructuran en 1 ECTS de clases teóricas, 0,5 ECTS de prácticas en el aula y 0,5 ECTS de prácticas de campo.

\section{Contenidos}

En este CIMA nos centraremos en mejorar la docencia de los Bloques 3 (Técnicas de análisis más utilizadas en el estudio de artefactos líticos) y 4 (Técnicas de análisis empleadas en el análisis de otros tipos de materiales) del programa de la asignatura, teniendo en cuenta los resultados esperados del aprendizaje, ante citados, los conceptos, procedimientos, aptitudes y actitudes que han de entrenarse en la asignatura, así como el punto de partida de los alumnos. Intentaremos, por tanto, estructurar los contenidos para dar cabida a los que consideramos fundamentales en el aprendizaje del alumno. La idea que subyace es la de recortar todo lo que es accesorio para quedarnos con lo estructurador.

Para ello, lo primero que realizaremos es una síntesis de los conceptos que creemos esenciales y que 
estructuran el Grado en Arqueología y la asignatura Análisis de Artefactos.

Conceptos estructuradores a nivel de Grado:

1. La Arqueología tiene 3 dimensiones: espacio, tiempo y forma.

2. La Arqueología es una disciplina materialista que trata con artefactos que son evidencia de acciones y conductas pasadas.

3. La inferencia en Arqueología se realiza a través del análisis de los artefactos y su contexto.

Conceptos estructuradores de la asignatura:

1. Análisis de artefactos supone la adquisición de la evidencia empírica acerca de las acciones y conductas pasadas.

2. Análisis de artefactos es la base empírica de la inferencia en Arqueología.

3. Existe una interrelación entre los datos empíricos y las dimensiones de la Arqueología.

4. Necesitamos huir de los tópicos y generar una base empírica de calidad para que la disciplina avance. Es, por tanto, necesario que se conozcan los principios básicos de las técnicas con las que adquirimos los datos.

La mayor parte de estos conceptos estructuradores del grado y de la asignatura entrenan aptitudes y actitudes. Serán por tanto estos los contenidos estructuradores de la asignatura.

Los contenidos que se van a desarrollar a nivel programático se detallan en la Figura 1.

Jornadas de Formación e Innovación Docente del Profesorado | № 2 (2019) Esta obra se distribuye con la licencia Creative Commons 


\section{CARLOS P. ODRIOZOLA}

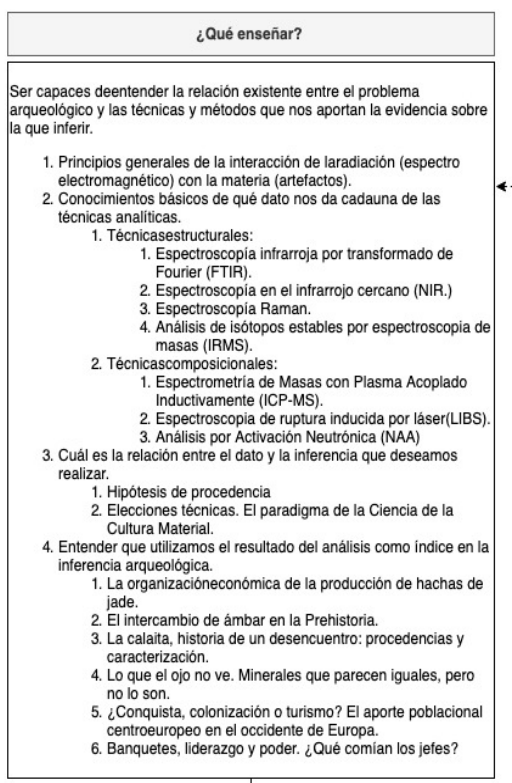

Contenidos

\section{Conceptual}

1. Conocer los principios básicos de algunas técnicas de análisis, terminologia utilizada y su aplicación al material análisis, terminoologico.

2. Primera toma de contacto con algunos de los equipos de análisis más comunes.

3. CG5- Que los estudiantes se familiaricen con los métodos y las técnicas de investigación de otras disciplinas que comparten el ambito de estudio de la Arqueologia desde
otras perspectivas y adquirir los rudimentos básicos de las otras pers
mismas.

\section{Actitudinal}

- CE4- Que los estudiantes aprendan a manejar criticamente los métodos y técnicas para recuperar el registro arqueologico e identificar, analizar e interpretar datos arqueologicos.

\section{Aptitudinal}

- CB3-Que los estudiantes tengan la capacidad de reunir e interpretar datos relevantes para emitir juicios que incluyan una reflexion sobre temas relevantes de indole social, cientifica o ética.

CB4- Que los estudiantes puedan transmitir información, ideas, problemas y soluciones a un público tanto especializado como no - CT4- Que los

diantes desarrollen la capacidad de análisis y sintesis de temas arqueologicos, desarrollando razonamiento critico y autocritico.

\section{Resultados del aprendizaje}

1. Conocimiento de los métodos y técnicas de obtención y elaboración de datos

2. Conocimiento de los fundamentos de las técnicas fisicoquimicas utilizadas en Arqueologia

3. Aprenderla correcta lectura e interpretación de los resultados de estas analiticas

4. Desarrollar una buena percepción del concepto de analitica y su alcance

Figura 1. Desarrollo programático de los bloques 3 y 4 de la asignatura en relación con los contenidos y resultados del aprendizaje programados en la Memoria de verificación.

Jornadas de Formación e Innovación Docente del Profesorado I № 2 (2019) 
Una vez diseñado el contenido programático y explorada la relación entre los contenidos consignados en la Memoria de Verificación y el programa de la asignatura aprobado para el curso 2018-2019 (Figura 1) se procede a organizar los contenidos en forma de mapa de contenidos (Figura 2).

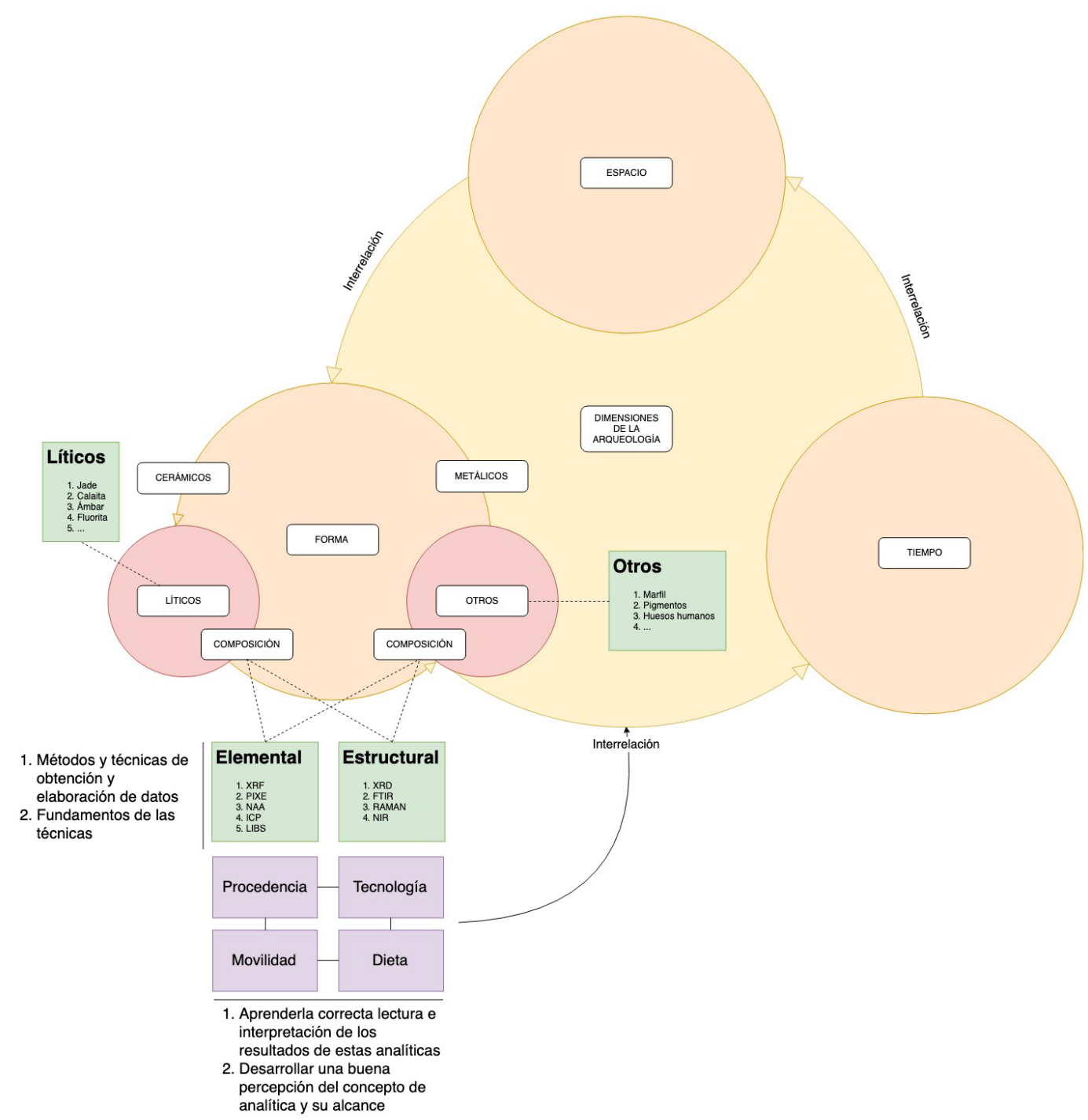

Figura 2. Mapa de contenidos.

Jornadas de Formación e Innovación Docente del Profesorado | № 2 (2019)

Esta obra se distribuye con la licencia Creative Commons

Reconocimiento-NoComercial-SinObraDerivada

4.0

Internacional (CC BY-NC-ND 4.0.) 


\section{Modelo metodológico y secuencia de actividades programada}

Actualmente la metodología reflejada en el proyecto docente del curso 2018-2019 es un modelo basado en la transmisión que pretende asemejarse a un modelo basado en la teoría y su aplicación a la práctica. Para ello utiliza una combinación de seminarios introductorios con clases prácticas de laboratorio, que persiguen dotar al alumnado de las habilidades mínimas necesarias para diseñar, implementar y realizar análisis instrumentales de artefactos arqueológicos. Pero, de facto, las clases prácticas no son más que clases transmisivas realizadas en otro entorno, el laboratorio.

En este CIMA pretendemos sustituir este modelo metodológico "tradicional" por un modelo metodológico "centrado en el estudiante", que practique una visión constructivista y evolutiva del conocimiento, donde el estudiante se sitúe en el centro del aprendizaje, trabajando en la propia construcción del aprendizaje y transformando el modelo actual de clase teórica en procesos que conduzcan desde la investigación individual y el trabajo colaborativo al conocimiento sensu Finkel (2008).

Se pretende que el alumno a través del planteamiento de problemas por parte del profesor tome conciencia de estos problemas y que a través de actividades diseñadas por el docente contraste ideas, llegando a proponer procedimentalmente alternativas conceptualmente lógicas, para a través de un proceso de investigación centrado en el contraste de los procedimientos desarrollados / planteados por sí mismo y los propuestos inicialmente, el alumno construya conocimiento de forma crítica y razonada usando la lógica y el método científico (Figura 3). 


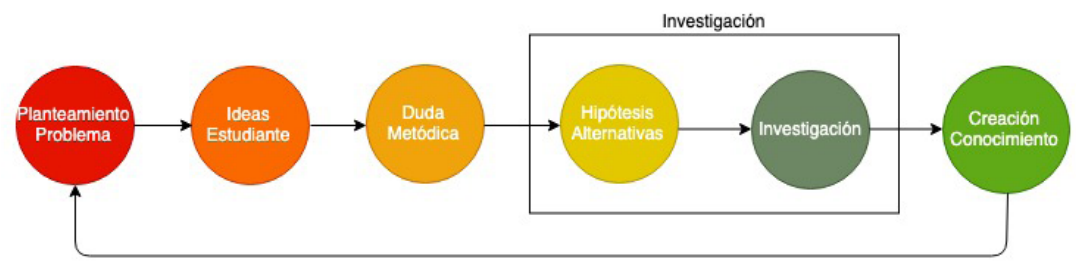

Figura 3. Representación del modelo metodológico mixto basado en la reelaboración de las ideas del estudiante y el principio de investigación.

Para favorecer el ajuste entre el modelo metodológico elegido y el aprendizaje o creación de conocimiento del alumnado, no sólo hay que diseñar una secuencia de actividades entendidas como "unidades de programación", sensu De Alba y Porlan (2016), que comiencen con el planteamiento de un problema y acaben en la construcción de conocimiento a través de una reestructuración del conocimiento / ideas previas del alumnado vía un proceso de investigación y contrastación, sino que deberemos ajustar los niveles de partida de los contenidos a aprender a los iniciales del alumnado siguiendo un modelo de construcción progresiva o "hipótesis de progresión" sensu García Díaz, Porlán y Navarro (2016).

La secuencia de actividades diseñada se recoge sintéticamente en la Figura 4.

Jornadas de Formación e Innovación Docente del Profesorado | № 2 (2019) Esta obra se distribuye con la licencia Creative Commons Reconocimiento-NoComercial-SinObraDerivada 


\section{Lectura y debate}

El pueblo Campaniforme, desde una visión eugenésica.

La invasión de los braquicéfalos.
Taller conceptual

El Campaniforme, aproximación a la

- interacción social: movilidad e

intercambio.

\section{Práctica grupal}

Identificación del artefacto (lítico) y $\rightarrow$ su materia prima.

Propuesta de metodologia.

Juego: demuestra que tu oponente miente.

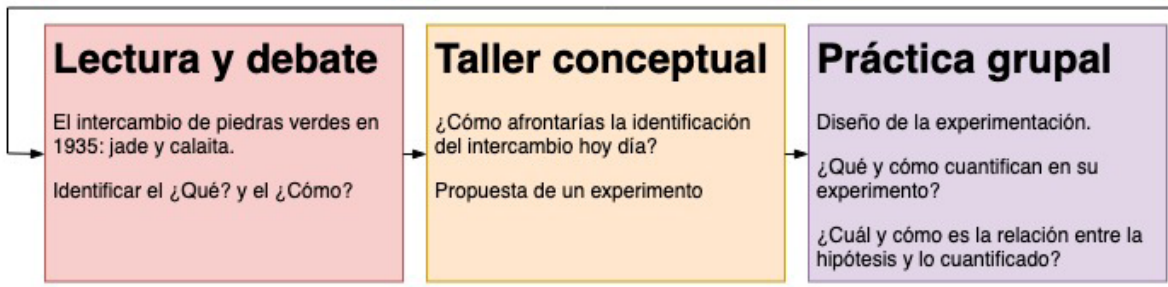

\section{Lectura y debate Lectura y debate Vídeo y debate}

Las dimensiones de la Arqueologia.

El ámbito y las preguntas en el nacimiento de la Arqueología moderna (Nueva Arqueologia).

Los grandes retos de la Arqueología en el siglo XXI.

Los métodos y técnicas actuales y su papel en el desempeño de la Arqueologia hoy.
Documental, acerca de un proyecto

$\rightarrow$ arqueológico actual (pigmentos).

¿Es la pregunta actual? ¿Y los métodos y técnicas?

¿Miente? ¡Demuéstralo!

\section{Píldora info.}

Estructura versus composición

¿Cuántos componentes químicos tiene un artefacto y cómo se estructuran?

\section{Vídeo}

La tabla periódica de los elementos químicos.

¿Qué es un elemento y qué diferencias tienen?

\section{Práctica grupal}

¿Qué técnicas de análisis se pueden - aplicar a materiales arqueológicos?

Haz un listado de técnicas y su aplicación.

Explica que cuantifica cada una.

\section{Vídeo y debate}

El espectro electromagnético (NASA)

¿Qué relación existe entre los

fenómenos fisicoquímicos y el problema arqueológico?

\section{Taller conceptual}

Diseño de experimentación para

$\rightarrow$ resolver un problema de la

Arqueología actual.

Teoria, método y su relación ¿Qué cuantificamos, cómo y para qué?

Figura 4. Secuencia de actividades estructuradas en unidades de programación.

Jornadas de Formación e Innovación Docente del Profesorado | № 2 (2019) Esta obra se distribuye con la licencia Creative Commons Reconocimiento-NoComercial-SinObraDerivada Internacional (CC BY-NC-ND 4.0.) 


\section{Aplicación del Ciclo de Mejora en el Aula}

La aplicación del CIMA se ha llevado a cabo entre los días 25 de marzo y 10 de abril de 2019 en un total de 20 horas lectivas, a razón de 6 horas semanales, en sesiones de 120 minutos diarias. El diseño contempla actividades a desarrollar en clase y en casa. En términos generales el ciclo se ha desarrollado según lo previsto, a excepción de alguna actividad puntual que estaba diseñada para realizar en clase y los alumnos tuvieron que desarrollarla en casa porque alguna de las actividades de la sesión ocupó más tiempo del previsto en el diseño.

Inicialmente, la actitud de los alumnos, cuando se les plantea que se va a implementar una innovación en la asignatura con el objetivo de mejorar la docencia y la propia asignatura, es positiva, aunque se aprecia cierto tono de escepticismo. A mitad del desarrollo del ciclo realicé un sondeo informal para conocer la acogida que estaba teniendo la metodología docente frente a una metodología "tradicional" de tipo transmisivo. Algunos de los pocos que han respondido ha sido para expresar satisfacción con comentarios como "así aprendo más". Sin embargo, una alumna muy reflexiva, ha criticado constructivamente que a veces los debates y discusiones se derivan hacia temas que no están estrictamente relacionados con la lección (parte culpa del moderador y parte culpa de los alumnos más activos que tienden a llevar el debate a su campo o zona de confort), proponiendo que haya un guion que guíe y centre el debate en los temas de aprendizaje planteados con la actividad. Cuestión que sin dudad adopté en siguientes sesiones, ya que además esas preguntas o temas centrales de la discusión ya están presentes en el diseño de las actividades de debate.

En términos generales, una de las mayores dificultades que he encontrado en el desarrollo del ciclo es ajustar las actividades al tiempo previsto, ya que generalmente el 
tiempo previsto es menor que el tiempo real de desarrollo de la actividad. En algunas ocasiones no me ha sido sencillo controlar el tiempo de debate o el de lectura con comentarios críticos. En unos casos porque los debates se han alargado más del tiempo previsto y en otros porque los estudiantes tenían más claras de lo que pensaba algunas cuestiones; en varias ocasiones me ha preocupado andar mirando a menudo el tiempo restante de clase, sobre todo en momentos en los que la clase estaba siendo más dinámica e interesante.

De entre las actividades programadas en el CIMA, quizás destaquen por su utilidad la combinación de ronda de preguntas acerca del concepto, debate y crítica de textos "viejos" que tratan el concepto desde una perspectiva procedimental e ideológica no actual que motive al estudiante a investigar en grupo, y que como resultado de esa investigación se reelaboren ideas resultando en la creación de nuevo conocimiento. Una especie de "taller conceptual" al estilo del propuesto por Finkel (2008) que busca retener la atención de los alumnos a través de temas atractivos (Bain, 2007). Por ejemplo, en la sesión 1 y en la 2 se trataba de desarrollar conceptualmente el punto 1 del programa a través de una ronda de preguntas sobre el concepto "campaniforme" y la lectura de un artículo de Thomas Bubner.

La lectura del artículo de Bubner les motivó sobremanera; se trata de un artículo racista y políticamente incorrecto, incómodo e impensable hoy en día. Durante el debate lo critican duramente por la ideología racista, pero ignoran la metodología. Ésta es exquisita, científica y perfectamente aplicada, aunque a su entender es imposible que la eugenesia sea defendible científicamente. El debate es acalorado y los alumnos parecen motivados y en cierta medida decididos a investigar como desmontar los argumentos del artículo científicamente. Consigo el objetivo de la actividad, motivarlos a investigar en grupos en casa en contra de la visión de Bubner, que el profesor hace 
propia en el debate juego del día siguiente. En el mismo, en general, se ha realizado una sintesis de los aspectos teóricos que traía cada grupo preparados con respecto al campaniforme, posteriormente han expuesto cómo los autores se enfrentan al problema a nivel metodológico y hemos comenzado el debate juego acerca de si la metodología planteada realmente servía para resolver el problema. Algunos de los alumnos han sido particularmente críticos ante determinadas metodologías y otros se han centrado en debatir acerca de la ideología que subyace detrás de las hipótesis, planteando que, si la ideología era reprobable, lo era también la hipótesis y la metodología. Otros defienden que si la metodología es correcta entonces el trabajo científicamente es correcto, aunque después se utilice para justificar ideas reprobables.

Al acabar esta combinación de actividades la sensación fue gratificante, los alumnos habían logrado el objetivo consignado en el CIMA. Sin embargo, esta fórmula no fue tan exitosa en otros casos, lo que es ciertamente debido a una no tan acertada selección del texto por parte del profesor; los textos en inglés que he utilizado han contado con un rechazo generalizado por estar en inglés no por su contenido y ello ha provocado el efecto contrario al deseado, una especie de desmotivación hacia la actividad.

Otra de las herramientas utilizadas ha sido el visionado de audiovisuales. El desarrollo de estas actividades ha tenido diferentes resultados. Cuando los videos eran breves, 2-3 minutos en formato reportaje o 5-7 en formato documental, tuvieron muy buena acogida, sin embargo, cuando los videos eran más largos, 18-20 minutos, en formato mixto reportaje-documental, la acogida no fue tan buena y algunos alumnos confesaron que habían desconectado o que no les había supuesto un aprendizaje significativo. Sin duda, en este último caso el problema no es sólo la duración o el formato del video sino la temática y la calidad en la producción del audiovisual. En este sentido 
es dificil encontrar buenos audiovisuales en castellano, y dada la mala acogida y resultado de los textos en inglés no me atreví a repetir. Sin duda, en cursos venideros buscaré audiovisuales de calidad en la producción y si están en inglés los subtitularé.

Con la firme intención de cubrir las horas destinadas a clases prácticas de laboratorio-campo se han realizado actividades que implican el trabajo por parte del alumnado en pequeños grupos con materiales arqueológicos reales. A cada grupo se les ha asignado un conjunto de materiales que a simple vista son muy parecidos y se les ha pedido que los clasifiquen en base a sus características, preferentemente en cuanto a los tipos de materias primas. Una vez realizada la clasificación deben compartirla con el resto de los grupos exponiendo en qué se basan para realizar esa clasificación; el resto de los grupos debe debatir con el grupo que expone acerca de lo adecuado metodológicamente de la clasificación, proponiendo alternativas en el caso de discrepancia con la clasificación. Una vez concluida la actividad el profesor les indica qué materiales tienen entre manos y se le asigna a cada grupo la tarea de investigar en casa cómo podrían realizar la clasificación usando procedimientos actuales y teniendo en cuenta lo que saben del material. Tras la puesta en común de esta investigación grupal los alumnos han conseguido entender qué procedimientos se aplican a qué material y porqué. La actividad ha tenido muy buena acogida y los alumnos han agradecido sobremanera poder trabajar con materiales arqueológicos reales en problemas que son directamente relacionables con la actividad profesional.

El último recurso docente usado en este CIMA ha sido el comentario crítico de noticias en prensa. De nuevo, como en el caso del artículo de Bubner, el éxito no está tanto en la actividad sino en la noticia en sí misma. En este caso la noticia trata sobre el Tholos de Montelirio, un tema de interés local y europeo, que ha sido bastante mediático los 
últimos años. Pero, afinando más en el porqué ha sido tan exitosa esta actividad, creo que confluyen varias circunstancias, la primera es que es un tema con el que están familiarizados y que han estudiado en el Grado, y la segunda es que la noticia tiene un título gancho que despierta inmediatamente el interés del que lo lee: "El secreto de la tumba. Montelirio: 20 sacerdotisas de la Sevilla prehistórica envenenadas con mercurio". La actividad ha sido un éxito, consiguiendo una alta participación, muchos alumnos habían buscado información complementaria que no se había dado en clase. El éxito es achacable a que la actividad se entregó por escrito y después trabajamos en el debate, el cual me fue más fácil de dirigir y orientar hacía los temas que debían tratarse colateralmente, procedencia, movilidad, cambio tecnológico...

Con respecto al desarrollo del CIMA en su conjunto, cabe destacar que en algunas sesiones la lectura de textos se ha realizado como trabajo en casa en vez de en clase, por haber ocupado otras actividades más tiempo del previsto. En estos casos se le ha pedido al alumno que realice un comentario guiado escrito evaluable, es decir, que exprese por escrito una valoración razonada de los aspectos que al día siguiente se van a comentar sobre el texto y que son de interés. He encontrado esta fórmula más efectiva que la del comentario y debate libre, ya que se fuerza a los alumnos a salir de su zona de confort y centrarse en evaluar y debatir los temas que tienen mayor conexión o son centrales a los conceptos que pretenden impartirse con ese texto.

Por otra parte, en algunas ocasiones el desarrollo de los debates ha necesitado de mi intervención, no sólo para moderar y guiar el debate hacia determinados conceptos, sino para evitar errores conceptuales, lo que ha llevado a que determinadas "píldoras de conocimiento" se hayan adelantado en el tiempo a lo inicialmente diseñado. En otras ocasiones, la modificación del orden de 
las actividades, en concreto de las píldoras informativas, viene motivado porque los alumnos han mostrado interés en profundizar en aspectos que se tenía previsto llevar a cabo en futuras sesiones.

\section{Evaluación del Ciclo de Mejora en el Aula}

Para evaluar el ciclo de mejora, me voy a fijar, sobre todo, en la supuesta evolución del aprendizaje de los estudiantes. Para ello voy a partir de la presentación de la situación de partida para valorar posteriormente los resultados del momento de llegada.

\section{Cuestionario inicial: estableciendo el nivel de partida}

La evaluación del nivel de conocimiento inicial del alumnado, al comenzar el CIMA, se realiza a través de un cuestionario en el que se pretende explorar las ideas básicas, déficits conceptuales, formas de razonar, actitudes, prejuicios... El diseño de este cuestionario pretende cubrir dos aspectos básicos, por un lado, el nivel de las competencias básicas y transversales al grado y, por otro lado, las competencias específicas y de contenidos del alumnado. Este segundo punto es importante ya que determinará el punto de inicio y de finalización a la hora de la adquisición de contenidos programados y el nivel final de los resultados del aprendizaje.

Parte de los contenidos se han introducido ya en asignaturas como Registro Arqueológico (troncal, 2- curso) o Arqueogeología y Geoquímica (optativa, 30 curso), por lo que es importante averiguar la base de partida, los déficits conceptuales y la base media de conocimiento con la que llegan los alumnos. Todo esto es tremendamente variable ya que depende de los docentes que hayan impartido las 
asignaturas de 20 y 30 y de si los alumnos han cursado la optativa, mayoritariamente o no. Las cuestiones planteadas en el cuestionario fueron las siguientes:

1. ¿Cuáles son las dimensiones de la Arqueología?

2. ¿Cuál es la relación y el papel (rol) del análisis de artefactos (técnica) con la Arqueología (teoría y método)?

3. ¿Considera a la Arqueología una ciencia forense?

4. ¿Cómo se construye la inferencia Arqueológica?

5. ¿Qué entiendes por análisis de artefactos en arqueología?

6. ¿Qué tipo de análisis (técnicas) conoces que se puedan aplicar a artefactos líticos?

7. ¿Qué tipo de análisis (técnicas) conoces que se puedan aplicar a otros tipos de artefactos que no sean líticos, metálicos o cerámicos?

8. En líticos y otros artefactos ¿cuáles considerarías?

9. ¿Consideras necesario para el desarrollo de la Arqueología profesional conocer principios básicos de otras disciplinas científicas (ciencias auxiliares de la Arqueología)?

10. ¿Conceptos como intercambio, especialización artesanal, cadena operativa tienen su base en el análisis de artefactos?

Las respuestas tanto del cuestionario inicial como final se han agrupado en categorías en función a las ideas expresadas por el alumnado. Se han establecido cuatro niveles en función a la relación entre la respuesta/s seleccionadas y el razonamiento dado, que se han ordenado en función de su nivel de complejidad.

Los niveles, a pesar de ser específicos para cada pregunta, se pueden resumir de forma genérica como sigue:

Nivel 1: Desconocen y no razonan la respuesta.

Nivel 2: Tienen cierta idea y no son capaces de razonar la respuesta. 
Nivel 3: Conocen la respuesta pero no son capaces de razonarla.

Nivel 4: Conocen la respuesta y son capaces de razonarla.

A nivel cuantitativo puede apreciarse que en el primer bloque (Figura 5) dedicado a evaluar el nivel base de las competencias a entrenar en esta asignatura, tanto de las básicas y generales como de las transversales, domina para las 4 primeras preguntas el nivel 1 , mientras que la quinta pregunta está dominada por un nivel 2.

Podemos interpretar que es necesario realizar actividades docentes dedicadas a fortalecer o complementar conceptos transversales al Grado, conceptos procedimentales, a luchar en contra de tópicos superados hace 50 años y a generar una actitud positiva hacia el contenido que se va a impartir en este bloque de la asignatura.

De los resultados de la pregunta 5 podemos extraer que la mayoría de los alumnos tienen cierta idea acerca de los conceptos que se van a tratar en este bloque de la asignatura, lo que es quizás derivado a que ya han cursado 40 horas de la asignatura.

Del análisis cuantitativo de las respuestas del segundo bloque del cuestionario (Figura 5), dedicado a evaluar el nivel base de las competencias específicas, así como de los contenidos y resultados del aprendizaje podemos extraer que el nivel base en cuanto a lo conceptual y lo procedimental es mayoritariamente escaso (nivel 1). Poco o nada han retenido de los contenidos conceptuales superados en las asignaturas de 2 ㅇ y 30 o curso del Grado. Fue por tanto necesario partir de cero en estos contenidos conceptuales y procedimentales para llegar a un nivel en el que comprendan los conceptos, el qué, cómo y para qué de del aprendizaje de estos conceptos y procedimientos en Arqueología, así como de su lógica. No podemos 
pretender llegar a que comprendan los fenómenos fisicoquímicos subyacentes al procedimiento si no son capaces de comprender el porqué, el para qué, el cómo y la lógica subyacente a esto.

\section{Cuestionario final: análisis de la evolución del aprendizaje}

Para evaluar la propia efectividad del CIMA, siguiendo las directrices recogidas en Rivero y Porlán (2017), y una vez establecido el nivel de partida de los alumnos y desarrollado el ciclo de mejora, se pasó a los estudiantes al terminar dicho ciclo el mismo cuestionario a fin de determinar la efectividad de la intervención llevada a cabo.

El proceso de evaluación de los conocimientos iniciales y finales se ha realizado sobre un total de 32 estudiante matriculados. Utilizando el mismo cuestionario inicial y los escalones establecidos para el cuestionario inicial, en el análisis comparado de ambos cuestionarios, inicial y final, se aprecia un incremento sostenido en las respuestas de todas las preguntas para ambos bloques del cuestionario (Figura 5).

En el primer bloque de preguntas destinadas a evaluar el nivel base de las competencias a entrenar en esta asignatura, tanto de las básicas y generales como de las transversales, se aprecia un crecimiento sostenido, pero no destacado. La mayoría de los alumnos alcanzan el tercer y cuarto escalón, mientras que en el cuestionario inicial se encontraban en el escalón 1 y 2 (Figura 5). Por otra parte, las preguntas del segundo bloque, destinadas a evaluar el nivel base de las competencias específicas, así como de los contenidos y resultados del aprendizaje muestran un crecimiento muy destacado. Si en el cuestionario inicial la mayoría de los alumnos se encontraban en el nivel 1, en el final se encuentran entre el 3 y 4 (Figura 5). 


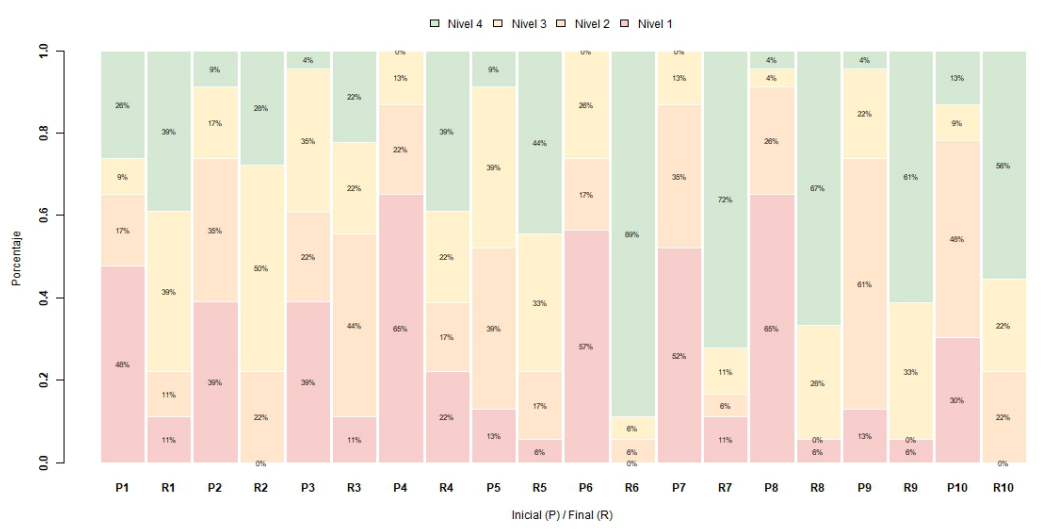

Figura 5. Resultado comparado para las preguntas del cuestionario inicial-final.

\section{Cuestiones a mantener y cambios a introducir}

En términos generales estoy satisfecho con la reestructuración de los contenidos, (conceptos, procedimientos, actitudes y aptitudes) que se enseñan. Habiendo reducido la cantidad de tiempo dedicado a los principios fisicoquímicos que hay detrás de cada procedimiento he sido capaz de ahondar en el concepto estructural que supone la relación entre artefacto e inferencia, el procedimiento analítico haciendo que los alumnos comprendan los distintos tipos de datos que obtienen de los procedimientos analíticos y su relación con la interpretación arqueológica, es decir, que sepan qué están midiendo y cómo se utiliza en Arqueología. Quizás este año, al ser el primero, se haya entrenado más la parte procedimental, actitudinal y aptitudinal que la conceptual en sí misma, por lo que en años venideros habrá que realizar ajustes en las actividades para llegar a compensar este déficit.

Si es cierto que las ideas planteadas en la asignatura están siendo bien acogidas por los alumnos, hay un porcentaje relativamente grande de alumnos en los que las ideas no arraigan y que han desarrollado una cierta 
resiliencia a las mismas, enrocándose en las ideas con las que llegaron a clase y no aceptando la intencionalidad de la enseñanza. Esto es sin duda fruto de que algunas de las actividades planteadas no ha sido lo eficaces que debieran, sobre todo la selección de audiovisuales y textos.

Hay que valorar positivamente que he tenido una asistencia media de 26 alumnos, de 32 matriculados, lo que supone una asistencia media del $81 \%$ de los matriculados, que es una cifra significativa para la asistencia habitual en el Grado en Arqueología.

A nivel metodológico me quedo con las actividades que mayor éxito han tenido en cuando a su capacidad para involucrar al alumno en su propio proceso de aprendizaje, textos polémicos y políticamente incorrectos hoy día como el de Bubner, comentarios de noticias de prensa cercanas que les puedan generar un vínculo emocional y provoque esa necesidad de investigar sobre algún concepto y el uso de talleres conceptuales que acaban en un juego por equipos, como la práctica con materiales reales.

Querría implementar para el curso que viene un concurso tipo trivial o quiz game en el que los alumnos participen por equipos a modo de sistema de evaluación; en este caso habría casilleros individuales y colectivos, las respuestas erróneas no puntuarían negativo y la calificación se establecería una vez que el primer equipo logra completar todos lo retos. El resto de los equipos quedará ordenado en base al número de ítems logrado en cada categoría habiendo tenido las mismas oportunidades de respuesta o más que el equipo que ha completado el reto.

A pesar de que se han impartido todos los contenidos programados inicialmente, muchas de las lecturas programadas para hacer en clase hubo que hacerlas en casa debido a que no he sabidor manejar bien los tiempos y se me han alargado demasiado los debates. Una solución a este 
problema es que las lecturas vengan hechas de casa y el alumno haya tenido que reflexionar y estructurar su pensamiento en los conceptos a tratar tras esa lectura ya que la lectura no es más que una excusa para que el alumno reflexione y cree conocimiento nuevo a través de la investigación comparada.

Con respecto a la evaluación del CIMA y el cuestionario, parece que 10 preguntas son muchas, ha llevado más tiempo del que había previsto, por lo que en una próxima ocasión las repensaré y condensaré en 506 preguntas.

Entre los principios didácticos que han guiado la experiencia docente -como al comienzo expresé- se encuentra un modelo metodológico que considero una mezcla entre el modelo basado en la reelaboración de las ideas del estudiante y el modelo basado en el principio de la investigación. Este modelo es un cambio radical pero tremendamente positivo con respecto al "tradicional", lo que se ha traducido en un incremento en la participación en clase de los estudiantes.

Por otro lado, el empleo del cuestionario inicial final es un buen barómetro a partir del cual acercarnos al nivel de los estudiantes y el resultado del aprendizaje.

Más allá de los cuestionarios para evaluar los conocimientos de los estudiantes en el CIMA o del modelo metodológico que planteo a los alumnos, me gustaría en paralelo para años venideros ajustar las actividades para cumplir con los preceptos TORA (Ham 1992), es decir, que las explicaciones de conceptos, procedimientos, actitudes o aptitudes sean temáticas, organizadas, relevantes y atractivas. Además, seguiré buscando textos, audiovisuales, artículos de prensa, etc. que conecten los conceptos con un tema de interés para el alumnado sensu Thorndyke (1977).

Jornadas de Formación e Innovación Docente del Profesorado | № 2 (2019) Esta obra se distribuye con la licencia Creative Commons Reconocimiento-NoComercial-SinObraDerivada 


\section{Referencias bibliográficas}

Bain, K. (2007). Lo que hacen los mejores profesores universitarios. Valencia, España: Publicacions de la Universitat de València.

De Alba, N. y Porlán, R. (2017). La metodología de enseñanza. En R. Porlán (Coord.), Enseñanza Universitaria. Cómo mejorarla (pp. 37-54). Madrid: Morata.

Finkel, D. (2008). Dar clase con la boca cerrada. Valencia: Publicacions de la Universitat de València.

García Díaz, E., Porlán, R. y Navarro, E. (2017). Los fines y los contenidos de la enseñanza. En R. Porlán (Coord.), Enseñanza universitaria. Cómo mejorarla (pp. 54-72). Madrid: Morata.

Ham, S. (1992). Environmental interpretation: A practical guide for people with big ideas and small budgets. Golden: North American Press.

Rivero, A. y Porlán, R. (2017). La evaluación de la enseñanza universitaria. En R. Porlán (Coord.), Enseñanza Universitaria. Cómo mejorarla (pp. 73-92). Madrid: Morata.

Thorndyke, P. W. (1977). Cognitive Structures in Comprehension and Memory of Narrative Discurse. Cognitive Psychology, 9(1), 77-110.

Jornadas de Formación e Innovación Docente del Profesorado | № 2 (2019) Esta obra se distribuye con la licencia Creative Commons 